\title{
Cross-scale Dynamo Action in Multiscale Plasma Turbulence
}

\author{
Jiquan LI and Yasuaki KISHIMOTO \\ Graduate School of Energy Science, Kyoto University, Uji, Kyoto 611-0011, Japan
}

(Received 12 July 2010 / Accepted 20 July 2010)

\begin{abstract}
The interplay mechanism between electromagnetic (EM) and electrostatic (ES) turbulence is explored using direct gyrofluid simulations of mixed resistive magnetohydrodynamic (MHD) and ion-scale microinstability. With an elucidation based on a minimal model, we propose a new concept of cross-scale dynamo action induced by microturbulence as the mechanism responsible for the interaction between multiscale ES and EM turbulence. The dynamo appears as a novel magnetic island seesaw oscillation pivoting around the singular surface. This island seesaw mechanism may be applicable to relaxation of mode locking in tokamaks to mitigate major disruptions, suggesting a promising nonlinear approach to plasma control.
\end{abstract}

(c) 2010 The Japan Society of Plasma Science and Nuclear Fusion Research

Keywords: multiscale interaction, direct simulation, microturbulence, MHD, dynamo, magnetic island

DOI: $10.1585 /$ pfr.5.031

MHD and microturbulence are common fluctuations in tokamaks and stellarators; they are important in plasma equilibrium, control, and confinement performance and have been studied for many decades. Electromagnetic (EM) MHD fluctuations are dominated by magnetic dynamics and reconnection. Microturbulence is characterized by electrostatic (ES) turbulence with flow dynamics. Renewed interest has arisen recently in the multiscale nonlinear interaction among them [1-9]. This is a natural concern in fusion devices. MHD perturbations, especially those with large magnetic islands, may modify the equilibrium configuration, likely affecting the microinstability, and island dynamics and magnetic reconnection may change in a turbulent environment with small-scale fluctuations. The interplay between ES microturbulence and the EM MHD mode should become prominent owing to highly complex nonlinearity, particularly in future fusion reactors such as ITER. It may further create new nonlinear dynamics.

Of particular interest are the interaction mechanism and energy-exchange channel between ES and EM turbulence with different scales. A typical EM environment including ES potential is described by the full MHD equation in many astrophysical bodies [10], in which dynamo action plays a key role in transforming kinetic energy into magnetic energy. In kinematic dynamo theory, the dynamo current and field are induced by a given stationary flow. This process might provide new insights into the abovementioned concerns in a multiscale dynamic system.

In this Rapid Communications, we report a novel result based on direct gyrofluid simulation of mixed resistive MHD and ion temperature gradient (ITG) driven turbulence. We propose the new concept of cross-scale dynamo action induced by microturbulence to understand the un-

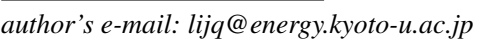

derlying interaction between multiscale ES and EM turbulence due to different instabilities. A promising application to plasma control is suggested.

Simulations are performed using an initial value code in a current sheet based on a five-field gyrofluid model [6]. The resistive tearing mode [11] and ITG instability [12] are consistently involved. The details of the physical model and simulation setting have been described in [6], which emphasized the oscillatory nature of zonal flow dynamics. Here, the focus is mainly on the island dynamics. A prominent magnetic island oscillation is commonly observed in the final nonlinear stage with a fully reconnected island (namely, with larger tearing instability parameter $\Delta^{\prime}[11]$ ), showing a dynamic quasi-steady state. A typical simulation with ion temperature gradient parameter $\eta_{\mathrm{i}}=2.0$ and resistivity $\eta=5 \times 10^{-4}$ is specified. The island oscillation is visualized by a movie of island evolution, and much more clearly, by snapshots of the dominant flux component $m=1$. The oscillation occurs as pivoting along the singular surface like a seesaw (referred to hereafter as an island seesaw). The averaged EM torque [13]

$$
T_{\mathrm{EM} y} \hat{z}=\iint_{x y} x \hat{x} \times(\vec{j} \times \vec{B})_{y} \mathrm{~d} x \mathrm{~d} y / L_{x} L_{y}
$$

exerted on the island by the fluctuating EM force in the $y$ direction increases dramatically and tends to oscillate in time in the quasi-steady state, as shown in Fig. 1 (a), synchronizing with the island seesaw. The EM torque and seesaw amplitude increase with increasing $\eta_{\mathrm{i}}$. For smaller $\Delta^{\prime}$, reconnection also occurs, but the island is saturated at small width, so the magnetic island remains static regardless of the ITG intensity.

To explore the island seesaw mechanism, we propose a minimal model consisting of the reduced MHD equations $[11,13]$ with the normalization for drift waves 

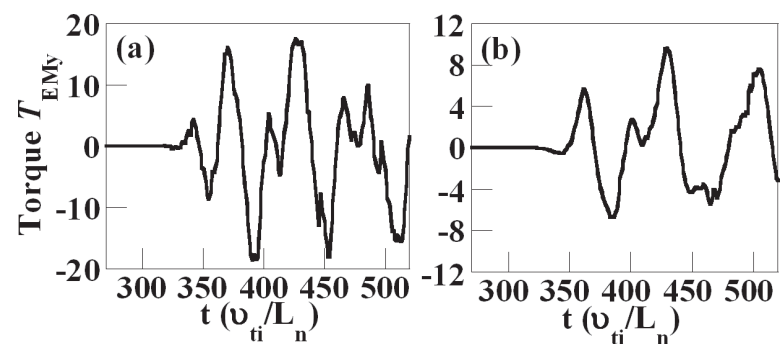

Fig. 1 Averaged EM torque (a) in direct simulation, (b) in modeling simulation.

$$
\begin{aligned}
& \partial_{\mathrm{t}} \nabla_{\perp}^{2} \phi=-\left[\phi, \nabla_{\perp}^{2} \phi\right]+[\psi, j], \\
& \beta \partial_{\mathrm{t}} \psi=-[\phi, \psi]+\eta j,
\end{aligned}
$$

and an independently evolving ITG eigenmode [12],

$$
\phi^{\mathrm{ITG}}\left(t, x, k_{y}^{\mathrm{ITG}}\right)=\hat{\phi}^{(n)}(x) \mathrm{e}^{-i \Omega t+i k_{y}^{\mathrm{ITG}} y} .
$$

The latter, representing microturbulence, is involved in the Poisson brackets through $\phi=\phi^{\mathrm{MHD}}+\phi^{\mathrm{ITG}}$. The eigenfunction $\hat{\phi}^{(n)}(x)=H^{(n)}(\sqrt{i \sigma} x) \exp \left(-i \sigma x^{2}\right)$ corresponds to the complex eigenvalue $\Omega$. $\sigma$ denotes the parametric dependence, and the Hermite function $H^{(n)}$ determines the radial parity of the ITG eigenmode. Using the kinematic dynamo theory [10], the level of $\phi^{\mathrm{ITG}}$ can be artificially chosen following Eq. (4) as a given flow component considering the ITG spectral features resulting from the inverse cascade in time and space.

Modeling simulations are performed using the same parameters and numerical setting as in Fig. 1 (a). An island seesaw similar to that in the direct simulations is observed only when $\phi^{\mathrm{TTG}}$ has radial even parity. The seesaw amplitude and oscillatory EM torque increase with increasing $\phi^{\mathrm{ITG}}$, showing the same dependence on the ITG mode as in the direct simulations. The seesaw oscillation is characterized by the real frequency $\Omega$ of the ITG mode. Note that in mixed MHD and ITG turbulence [6], the ITG spectrum connects smoothly with the MHD, showing that the ITG energy spectra are widely scattered because of nonlinear coupling. Here, we may assume an effective ITG mode to represent the whole turbulence. One multifold scan of $\Omega, k_{y}^{\mathrm{ITG}}$, and the ITG amplitude in the modeling simulation shows a best-matching eigenvalue $k_{y}^{\mathrm{ITG}}=0.3$ and $\Omega / \omega_{*} \approx 0.08$. The corresponding $T_{\mathrm{EM} y} \hat{z}$ is plotted in Fig. 1 (b), exhibiting similar behavior as in Fig. 1 (a), but with smaller amplitude because the single mode was selected. A full reconnection occurs, so the island behaves like a balloon floating in the air. The underlying mechanism may be understood through the new concept of crossscale dynamo action introduced here.

In Eq. (3), the magnetic flux responds to the interplay of the microscale $\phi^{\mathrm{ITG}}$ through the induction term to create a dynamo current component $j_{\mathrm{D}}$. The MHD flow $\phi^{\mathrm{MHD}}$ is influenced mainly by the Maxwell stress in Eq. (2) due to the dynamo components (simulations have shown
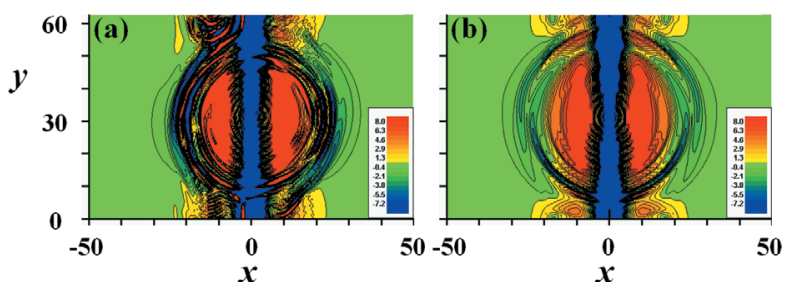

Fig. 2 Snapshots of current distribution in modeling simulations with radial (a) even-parity and (b) odd-parity ITG modes.

that the Reynolds stress is negligible). Note that the tearing parity is characterized by radially odd potential, denoted by $\phi^{\mathrm{MHD}}(x) \propto \sin \left(k_{x}^{\mathrm{MHD}} x\right)$, and even flux $\psi^{\mathrm{MHD}}(x) \propto$ $\cos \left(k_{x}^{\mathrm{MHD}} x\right)$ as well as even current. Any nonlinear interaction with external flows may possibly break the MHD symmetry. If an even $\phi^{\mathrm{ITG}}(x) \propto \cos \left(k_{x}^{\mathrm{ITG}} x\right)$ is applied, the dynamo current arising from $j_{\mathrm{D}} \propto \eta^{-1}\left[\phi^{\mathrm{ITG}}, \psi^{\mathrm{MHD}}\right]$ and the dynamo flux $\psi_{\mathrm{D}}^{\mathrm{MHD}}$ should have an odd parity based on the trigonometric function identity. The MHD potential and vorticity then involve an induced component $\phi_{\mathrm{D}}^{\mathrm{MHD}}$ and $\nabla_{\perp}^{2} \phi_{\mathrm{D}}^{\mathrm{MHD}}$ with radially even symmetry from Eq. (2), opposed to the tearing parity. The even parity of $\phi_{\mathrm{D}}^{\mathrm{MHD}}$ signifies a positive feedback of $\phi^{\mathrm{ITG}}$, showing a cross-scale dynamo action. The dynamo here is induced by a dynamic potential due to microturbulence, qualitatively different from those in kinematic dynamo theory [10]. The breaking of the MHD current symmetry is visualized in Fig. 2 (a). If a radial odd parity $\phi^{\mathrm{ITG}}$ is applied, all field components induced by the cross-scale dynamo action maintain the same tearing parity, as shown in Fig. 2(b). The EM torques, $T_{\mathrm{EM} y} \hat{z}$, on both sides of the singular surface have the same magnitude and almost cancel out each other owing to their opposed directions. Now it becomes clear that a dynamo current with only a radial odd-parity component may produce a non-zero oscillatory EM torque $T_{\mathrm{EM} y} \hat{z}$ to drive the island seesaw.

In conclusion, a new concept of cross-scale dynamo action induced by microturbulence is shown to be responsible for an island seesaw oscillation pivoting around the singular surface in multiscale ES and EM turbulence. The island seesaw may not only demonstrate a new energyexchange channel between ES and EM turbulence due to different instabilities, but also can likely be applied to relaxation of mode locking in tokamaks to mitigate major disruptions, suggesting a promising nonlinear approach to plasma control.

This work was supported by the Grant-in-Aid from JSPS (No. 19560828 and No. 21340171).

[1] C.J. McDevitt et al., Phys. Plasmas 13, 032302 (2006).

[2] Z.X. Wang et al., Phys. Plasmas 16, 060703 (2009).

[3] M. Yagi et al., Nucl. Fusion 45, 900 (2005).

[4] A. Ishizawa et al., Nucl. Fusion 15, 084504 (2008).

[5] F. Militello et al., Nucl. Fusion 15, 050701 (2008).

[6] J. Li et al., Nucl. Fusion 49, 095007 (2009). 
[7] T. Gorler et al., Phys. Rev. Lett. 100, 185002 (2008).

[8] Z.X. Wang et al., Phys. Rev. Lett. 103, 015004 (2009).

[9] M. Muraglia et al., Phys. Rev. Lett. 103, 145001 (2009).

[10] S.M. Tobias, Phil. Trans. R. Soc. Lond. A 360, 2741
(2002).

[11] N.F. Loureiro et al., Phys. Rev. Lett. 95, 235003 (2005).

[12] J. Li et al., Phys. Plasmas 5, 959 (1998).

[13] R. Fitzpatrick, Nucl. Fusion 33, 1049 (1993). 